of minority participants in the CLSA as they reach older ages will be essential to better understanding the relationship between the social determinants of health and aging in Canada.

\section{THE NEED TO APPEAR HEALTHY: CONCEALMENT OF CHRONIC ILLNESS, PRIVACY, AND SELF- SUFFICIENCY IN NIGERIA}

K. Mahmoud, University of Kansas

Among the negative effects of chronic diseases, the selfperceptions and the self-confidence of chronically ill persons deserve more research. This study explored how such persons dealt with the physical, mental and emotional changes brought about by the onset of chronic disease. The specific focus here was the role of social support networks in older patients' emotional coping. This qualitative study was conducted in two state-owned medical institutions in the northcentral part of Nigeria. In-depth interviews were conducted among 19 purposively selected, chronically ill persons aged 50 years and over who were receiving clinical care. This study revealed that except in extremely dire circumstances, older people with chronic conditions preferred to keep knowledge of their conditions strictly within their close family circles. It is almost a taboo to inform community members, friends and religious groups about one's chronic health difficulties. Reasons for the need to appear healthy to others might have stemmed from the fear of being discriminated against and attempts to maintain some level of normalcy when interacting with others. Moreover, social networks could also have a negative influence on older persons' emotional wellbeing. For example, many of the respondents received negative comments about their physical appearances. These statements resulted in participants having low self-esteem about their body images and consequently affected their participation in social activities. Thus, the supportiveness of social networks cannot be assumed. Outside of close family, social networks appear to be inadequately equipped to understand some of the sensitivities that chronically ill older persons struggle with.

\section{THE RELATIONSHIP BETWEEN WORK-LIFE BALANCE AND RETIREMENT PLANNING AMONG EMPLOYEES IN TAIWAN}

W. Hsu, S. Hu, National Cheng Kung University

Purpose This study constructed work-life balance variable as a predictor of retirement planning, and examined the relationship and interaction effect between work-life balance and retirement planning. Method This study analyzed 1362 samples aged more than 45 years old from the survey data of "Employees Retirement planning Needs Assessment" in Taiwan. Retirement planning score was measured by the scale developed by Noone et.al in 2010. Work-life balance was measured using the engagement of life items and work satisfaction questionnaire. The scores were computed and divided into 4 types: (1) work-life balance, (2) life imbalance, (3) work imbalance, and (4) work-life imbalance. Descriptive statistics, Chi-Square, T-test and multiple regressions were used in this study. Result The results showed that $22.4 \%$ of the employees had work-life balance, $35.8 \%$ had life imbalance, $14.0 \%$ had work imbalance and $26.2 \%$ had work-life imbalance. Comparing employees in the group of work-life imbalance group, those who in the groups of work-life balance and work imbalance had significantly higher retirement planning score $(\beta=5.5$ and 1.51 , respectively). The life imbalance group have the same retirement planning score as the work-life imbalance but did not differ significantly. In addition, engagement of life and work satisfaction had significant interaction effect to retirement planning. Discussion According to the results, employees who have work-life balance tend to have better retirement planning. The government should encourage employees to actively engage in life, such as domesticity, social participation, care, cultivate interest, among others. Higher engagement in the life domain can significantly promote employees retirement planning.

\section{THE RELATIONSHIPS BETWEEN KOREAN ADULT CHILDREN'S OUTCOMES AND THEIR PARENTS' PSYCHOLOGICAL WELLBEING}

M. Lim 1 , H. Jun' 2 1. Department of Child and Famliy Studies, Yonsei University, Seoul, 03722, Korea, 2. Yonsei University, Seoul, Republic of Korea

Guided by a life course perspective, the purpose of this study is to examine the linkages between adult children's outcomes for the transition to adulthood (employment, marital, and coresidence status) and their parents' psychological wellbeing, as well as whether these associations are similar for parental income. Regression models were estimated using data from 2,596 parents whose youngest child was at least 40 years old in the 2012 (the 4th wave) KLOSA (Korean Longitudinal Study of Ageing). Sons' employment and marital status and daughters' marital status (excluding children's coresidence status) was significantly associated with their parents' levels of wellbeing. Moreover, parents' income moderated the associations between children's outcomes and the level of their parents' life satisfaction. Unemployed sons and single sons and daughters jeopardized the life satisfaction of their mothers with low income, but not other subgroups, and coresidence with sons decreased the life satisfaction of fathers with high income, but not those with low income. In line with how the results suggest that parental psychological outcomes regarding adult children's circumstances may be different depending on income, this study has implications for intergenerational relationships in the sociocultural context. These findings also imply that parents may have different views about norms regarding the transition to adulthood depending on their economic backgrounds. In sum, based on the life course perspective and a stress process model, this study provides a comprehensive understanding of how adult children and family structural factors may contribute to individuals' wellbeing in old age.

\section{THINKING ABOUT THE END OF LIFE WHEN IT IS NEAR: A COMPARISON OF GERMAN AND PORTUGUESE CENTENARIANS}

D. Jopp ${ }^{1}$, K. Boerner ${ }^{2}$, K. Kim² ${ }^{2}$ A. Butt' ${ }^{2}$, O. Ribeiro ${ }^{3}$, L. Araujo ${ }^{4}$, C. Rott $^{5}, 1$. University of Lausanne, 2. University of Massachusetts Boston, 3. University of Aveiro \& University of Porto - CINTESIS, 4. CINTESIS; ESEV. IPV, 5. Heidelberg University

Centenarians approach the end of their lives with certainty. Yet, little is known about their thoughts about the end 
of life (EOL). Comparing centenarians from Germany and Portugal, this study examined common thinking of and planning for the EOL among centenarians, and whether views on EOL are shaped by cultural contexts and individual characteristics. Centenarians from two larger population-based centenarian studies (87 German and 128 Portuguese) responded to five questions regarding their views on EOL. Using Latent Class Analyses, we identified patterns of EOL thoughts and examined differences in country and individual characteristics by the derived patterns. A significant portion of centenarians in both countries reported not thinking about the EOL, not believing in the afterlife, and not having made EOL arrangements; perceiving the EOL as threatening and longing for death were less commonly endorsed. LCA identified three latent patterns of EOL thoughts: Class 1 (EOL thoughts, EOL arrangements, and afterlife beliefs); Class 2 (EOL arrangements and afterlife beliefs); and Class 3 (overall low). The proportion of Portuguese centenarians was higher in Class 1 , whereas the proportion of German centenarians was higher in Class 2 and 3. Class membership was also related to centenarians' demographic, social, and health characteristics. In sum, findings indicate that despite closeness to death, centenarians do not necessarily think about and/or prepare for the EOL. Given that lack of EOL planning can result in poorer EOL quality, enhancing communication among centenarians, family, and health care professionals seems imperative.

\section{UGANDAN GRANDPARENT-CAREGIVERS: CONSEQUENCES OF CAREGIVING AND QUALITY OF LIFE IN THE HIV/AIDS ERA}

\section{S. Matovu, M. Wallhagen, University of California, San Francisco}

In this manuscript, we seek to highlight the consequences of caregiving and their impact on the health and overall quality of life of Ugandan grandparent-caregivers. Over the past two decades, the number of studies investigating grandparental caregiving provided to children affected by HIV/ AIDS in sub-Saharan Africa has gradually increased. With the sustained loss of lives due to AIDS, older adults are continuing to bear the burden of caring for children affected by the epidemic, often with very limited resources. Despite the acknowledgement of the elderly as the backbone and safety net of the African family in this HIV/AIDS era, very limited research has been conducted to explore the impact of this burden on the caregivers' mental health, physical wellbeing and overall quality of life. Thirty-two participants were recruited from urban and rural areas in Uganda and interviewed using a qualitative approach, specifically grounded theory methodology. The narratives generated from the semistructured and one-on-one interviews were audio-recorded, transcribed and analyzed using both open and axial coding as well as reflexive and analytic memoing congruent with the methodology. Descriptions of physical, financial and emotional caregiver burden were reported. Additionally, our study findings uniquely explored the impact of the perceived burden on their health and overall quality of life; and provided an explanatory model of the caregiving experience. Therefore, the study findings provide a foundation upon which clinicians, researchers and policy makers can design and implement effective interventions needed to improve the health and quality of life of grandparent-caregivers.

\section{WHO AMONG JAPANESE EMPLOYEES PREPARES} WELL FOR LIFE AFTER RETIREMENT?

K. Katagiri ${ }^{1}$, T. Onze ${ }^{2}$, 1. Kobe University, 2. Research Institute for Culture, Energy and Life, Osaka Gas

The life expectancy of Japanese people is one of the longest in the world. Although most Japanese companies still set the retirement age at 60 , through a recent amendment of the law, people can continue to work until the age of 65 with different work conditions before retirement. As the lifetime employment system was popular until recently and the organization culture of companies reflects a vertically-structured society, the Japanese are not accustomed to making plans and decisions about their careers. Little is known on this topic in Japan. This study examines those who plan well for their lives after retirement. An internet survey was conducted in Tokyo and the Osaka metropolitan area in 2016 by the Research Institute for Culture, Energy and Life, Osaka Gas. The sub-sample consisted of 924 people, aged 40-80 years. The result revealed that only a low percentage $(27 \%)$ of people in their fifties had planned for life after retirement. A logistic regression analysis was also conducted, in which demographic variables, social activity variables, social relations, and views of life were considered. People who were older, richer, single, house owners, participating volunteers, or those who had hobbies and valued their own way of life were more likely to have a definite plan after retirement. We observed no sex difference. Workaholics were at a higher risk of ill-preparation. The study therefore implies that an active private life other than work is necessary to sustain a long life after retirement.

\section{WIDOWHOOD AND MORTALITY RISK OF OLDER PEOPLE IN RURAL CHINA: DO GENDER AND LIVING ARRANGEMENT MAKE A DIFFERENCE?}

H. Liu ${ }^{1}$, Q. Jiang ${ }^{2}$, M. Feldman ${ }^{3}, 1$. Xi'an Jiaotong university, 2. Institute for Population and Development Studies, $X i$ an Jiaotong University, 3. Morrison Institute for Population and Resource Studies, Stanford University

Objectives: Increased mortality after spousal bereavement has been observed in many populations. Few studies have investigated the widowhood effect in a traditional culture where the economy is underdeveloped. In this study, we assessed whether the widowhood-associated excess mortality exists and differs by gender and living arrangement in rural China. Methods: The data used in this longitudinal study come from the survey "Well-being of Elderly Survey in Anhui Province (WESAP)", which was conducted every three years between 2001 and 2015 in rural townships of Anhui province. Excluding cases with missing values and restricting the sample to respondents who were married or widowed with adult children at baseline and in follow-up, analyses were carried out on 2,471 adults aged 60 and above. Cox regression was applied to examine the effects. Results: Spousal loss decreased mortality for older rural Chinese and there was a gender difference in this effect. Analyses also show that living with adult children after spousal loss played a protective role in reducing the risk of older men's death, though it tended to increase older men's mortality risk in general. Conclusion: Our findings suggest that the widowhood effect is culturespecific and spousal loss reduces rather than increases the mortality risk of rural elders in China, which implies that 